Acta Crystallographica Section E

Structure Reports

Online

ISSN 1600-5368

\section{4-Benzyl-4-methylmorpholinium hexafluorophosphate}

\section{Qiu-hong Su, ${ }^{\mathrm{a}}$ Hong-Jun Zang, ${ }^{\mathrm{a} *}$ Tian-lin $\mathrm{Xu}{ }^{\mathrm{b}}$ and Yuan-Lin Ren ${ }^{b}$}

aDepartment of Enviromental and Chemistry Engineering, Tianjin Polytechnic University, State Key Laboratory of Hollow Fiber Membrane Materials and Processes, Tianjin 300160, People's Republic of China, and ${ }^{\mathbf{b}}$ Department of Textiles, Tianjin Polytechnic University, State Key Laboratory of Hollow Fiber Membrane Materials and Processes, Tianjin 300160, People's Republic of China

Correspondence e-mail: chemhong@126.com

Received 14 September 2010; accepted 26 September 2010

Key indicators: single-crystal X-ray study; $T=113 \mathrm{~K}$; mean $\sigma(\mathrm{C}-\mathrm{C})=0.003 \AA$; disorder in main residue; $R$ factor $=0.049 ; w R$ factor $=0.114$; data-to-parameter ratio $=15.9$.

In the title compound, $\mathrm{C}_{12} \mathrm{H}_{18} \mathrm{NO}^{+} \cdot \mathrm{PF}_{6}{ }^{-}$, the asymmetric unit consists of two cation-anion pairs. The six $\mathrm{F}$ atoms of one anion are disordered over two sets of sites in a 0.592 (6):0.408 (6) ratio. The morpholinium rings adopt chair conformations.

\section{Related literature}

Ionic liquids based on the morpholinium cation are favored becaused of their low cost, easy synthesis, and electrochemical stability, see: Kim et al. (2006).

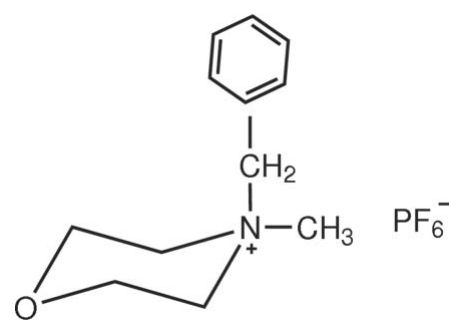

\section{Experimental}

Crystal data

$$
\begin{aligned}
& \mathrm{C}_{12} \mathrm{H}_{18} \mathrm{NO}^{+} \cdot \mathrm{PF}_{6}{ }^{-} \\
& M_{r}=337.24 \\
& \text { Triclinic, } P \overline{1} \\
& a=9.7268(14) \AA \\
& b=10.7183(16) \AA \\
& c=14.537(2) \AA \\
& \alpha=104.307(5)^{\circ} \\
& \beta=96.816(8)^{\circ}
\end{aligned}
$$

$\gamma=95.633(7)^{\circ}$

$V=1445.3(4) \AA^{3}$

$Z=4$

Mo $K \alpha$ radiation

$\mu=0.26 \mathrm{~mm}^{-1}$

$T=113 \mathrm{~K}$

$0.22 \times 0.14 \times 0.12 \mathrm{~mm}$

\section{Data collection}

Rigaku Saturn diffractometer

Absorption correction: multi-scan (CrystalClear; Rigaku/MSC, 2005)

$T_{\min }=0.946, T_{\max }=0.970$

Refinement

$R\left[F^{2}>2 \sigma\left(F^{2}\right)\right]=0.049$

$w R\left(F^{2}\right)=0.114$

$S=1.03$

6387 reflections

401 parameters

84 restraints

$\mathrm{H}$-atom parameters constrained

$\Delta \rho_{\max }=0.53 \mathrm{e} \AA^{-3}$

$\Delta \rho_{\min }=-0.40 \mathrm{e}^{-3}$

Data collection: CrystalClear (Rigaku/MSC, 2005); cell refinement: CrystalClear; data reduction: CrystalClear; program(s) used to solve structure: SHELXS97 (Sheldrick, 2008); program(s) used to refine structure: SHELXS97 (Sheldrick, 2008); molecular graphics: SHELXTL (Sheldrick, 2008); software used to prepare material for publication: SHELXTL.

The authors thank the Tianjin Natural Science Foundation (07JCYBJC02200) and (10JCZDJC22200) for financial support.

Supplementary data and figures for this paper are available from the IUCr electronic archives (Reference: JH2209).

\title{
References
}

Kim, K. S., Choi, S., Cha, J. H., Yeon, S. H. \& Lee, H. (2006). J. Mater. Chem. 16, 1315-1317.

Rigaku/MSC (2005). CrystalClear. Rigaku/MSC, The Woodlands, Texas, USA Sheldrick, G. M. (2008). Acta Cryst. A64, 112-122. 


\section{supporting information}

Acta Cryst. (2010). E66, o2695 [doi:10.1107/S1600536810038432]

\section{4-Benzyl-4-methylmorpholinium hexafluorophosphate}

\section{Qiu-hong Su, Hong-Jun Zang, Tian-lin Xu and Yuan-Lin Ren}

\section{S1. Comment}

Room-temperature ionic liquids (RTILs) consist of organic cation and anion which exist in liquid state at room temperature or below $100{ }^{\circ} \mathrm{C}$. They are widely used in various fields of electrochemistry and chemistry because of their unique properties such as nonvolatility and nonflammability. In particular, ILs based on the morpholinium cation are favored becaused of their low cost, easy synthesis, and electrochemical stability (Kim et al., 2006). So far, only a few crystallographic studies have been performed on salts. We report here a new example structure of this class.

The molecular structure of (I) is shown in Fig. 1. For the title compound two crystallographically independent molecules are present in the asymmetric unit of the cell. The morpholine unit adopts a chair conformation. Disorder model was introduced for the anion, in which the six fluorine atoms are all disordered over two positions. The bond distances and angles in the cation are normal within experimental error.

\section{S2. Experimental}

To a magnetically stirred solution of the 4-benzyl-4-methylmorpholinium chloride ( $2.29 \mathrm{~g}, 10 \mathrm{mmol})$ in acetonitrile (20 $\mathrm{ml}$ ) was added potassium hexafluorophosphate $(1.86 \mathrm{~g}, 10 \mathrm{mmol})$. The mixture was stirred at room temperrature for $72 \mathrm{~h}$, and the $\mathrm{KCl}$ filtered from the reaction mixture. The solvent was removed under reduced pressure. The residue was washed by ether and then recrystallized from hot ethanol to afford the product. A single-crystal was obtained by slow evaporation of a EtOH solution.

\section{S3. Refinement}

The $\mathrm{H}$ atoms bonded to $\mathrm{C}$ atoms were included in the refinement in the riding model approximation, with $\mathrm{C}-\mathrm{H}=0.93-$ $0.97 \AA$ and $U_{\text {iso }}(\mathrm{H})=1.2 U_{\text {eq }}(\mathrm{C}$ atom $)$. For the $\mathrm{H}$ atoms attached to $\mathrm{C}$ atoms of methyl groups, their $U_{\text {iso }}(\mathrm{H})=1.5 U_{\text {eq }}(\mathrm{C})$. 


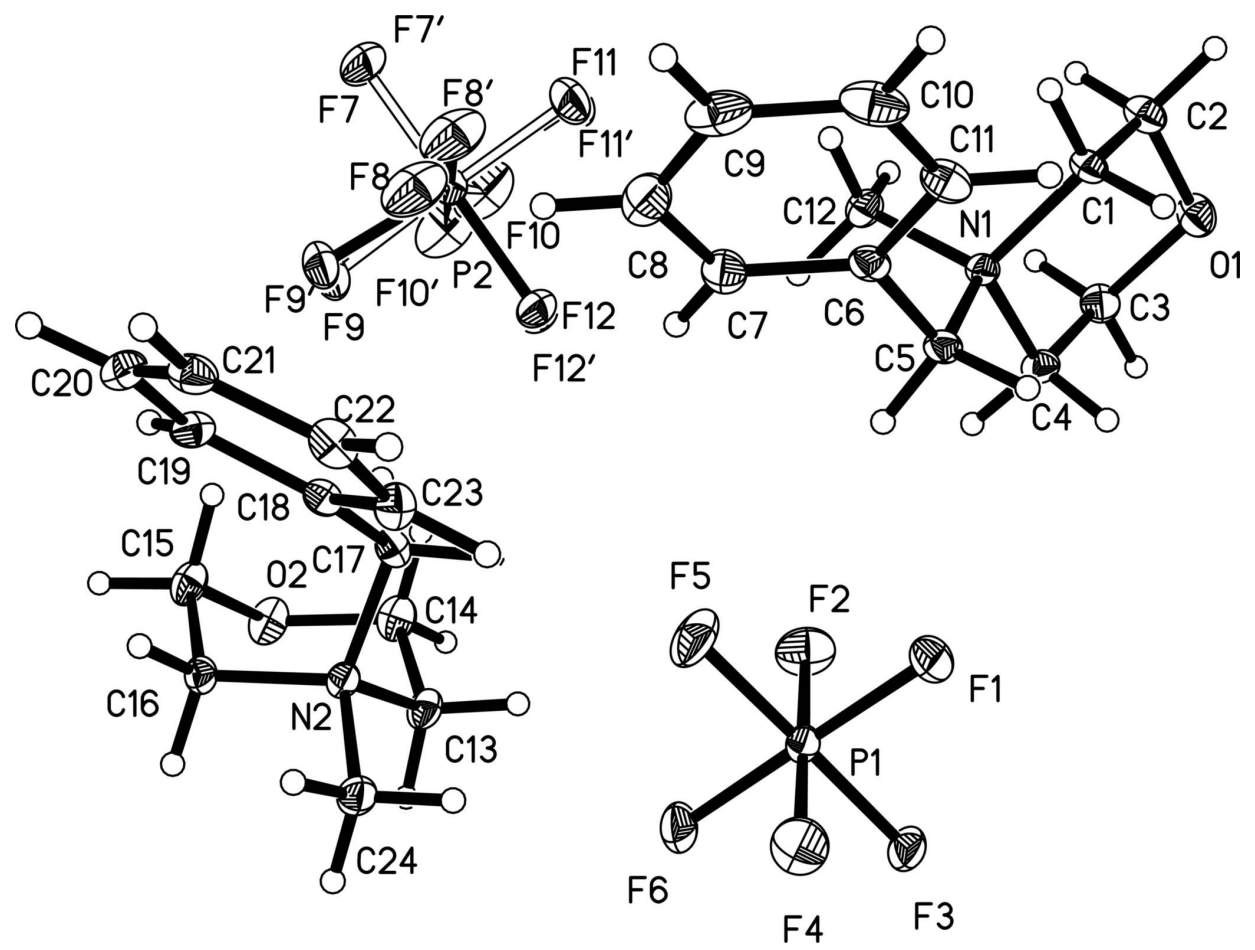

\section{Figure 1}

A view of the Structure of (I), Showing the atom-numbering scheme. Dispacement ellipsoids are drawn at the $30 \%$ probability level.

\section{4-Benzyl-4-methylmorpholinium hexafluorophosphate}

\section{Crystal data}

$\mathrm{C}_{12} \mathrm{H}_{18} \mathrm{NO}^{+} \cdot \mathrm{PF}_{6}^{-}$

$M_{r}=337.24$

Triclinic, $P \overline{1}$

$a=9.7268(14) \AA$

$b=10.7183(16) \AA$

$c=14.537(2) \AA$

$\alpha=104.307(5)^{\circ}$

$\beta=96.816(8)^{\circ}$

$\gamma=95.633(7)^{\circ}$

$V=1445.3(4) \AA^{3}$

\section{Data collection}

Rigaku Saturn diffractometer

Radiation source: rotating anode

Confocal monochromator

Detector resolution: 14.63 pixels $\mathrm{mm}^{-1}$

$\omega$ scans

Absorption correction: multi-scan

(CrystalClear; Rigaku/MSC, 2005)

$T_{\min }=0.946, T_{\max }=0.970$
$Z=4$

$F(000)=696$

$D_{\mathrm{x}}=1.550 \mathrm{Mg} \mathrm{m}^{-3}$

Mo $K \alpha$ radiation, $\lambda=0.71070 \AA$

Cell parameters from 4804 reflections

$\theta=1.5-27.2^{\circ}$

$\mu=0.26 \mathrm{~mm}^{-1}$

$T=113 \mathrm{~K}$

Block, colorless

$0.22 \times 0.14 \times 0.12 \mathrm{~mm}$

17415 measured reflections

6387 independent reflections

4730 reflections with $I>2 \sigma(I)$

$R_{\text {int }}=0.043$

$\theta_{\max }=27.2^{\circ}, \theta_{\min }=1.5^{\circ}$

$h=-12 \rightarrow 12$

$k=-13 \rightarrow 13$

$l=-18 \rightarrow 18$ 


\section{Refinement}

Refinement on $F^{2}$

Least-squares matrix: full

$R\left[F^{2}>2 \sigma\left(F^{2}\right)\right]=0.049$

$w R\left(F^{2}\right)=0.114$

$S=1.03$

6387 reflections

401 parameters

84 restraints

Primary atom site location: structure-invariant direct methods

Secondary atom site location: difference Fourier map

\section{Special details}

Geometry. All e.s.d.'s (except the e.s.d. in the dihedral angle between two 1.s. planes) are estimated using the full covariance matrix. The cell e.s.d.'s are taken into account individually in the estimation of e.s.d.'s in distances, angles and torsion angles; correlations between e.s.d.'s in cell parameters are only used when they are defined by crystal symmetry. An approximate (isotropic) treatment of cell e.s.d.'s is used for estimating e.s.d.'s involving 1.s. planes.

Refinement. Refinement of $F^{2}$ against ALL reflections. The weighted $R$-factor $w R$ and goodness of fit $S$ are based on $F^{2}$, conventional $R$-factors $R$ are based on $F$, with $F$ set to zero for negative $F^{2}$. The threshold expression of $F^{2}>\sigma\left(F^{2}\right)$ is used only for calculating $R$-factors(gt) etc. and is not relevant to the choice of reflections for refinement. $R$-factors based on $F^{2}$ are statistically about twice as large as those based on $F$, and $R$ - factors based on ALL data will be even larger.

Fractional atomic coordinates and isotropic or equivalent isotropic displacement parameters $\left(\AA^{2}\right)$

\begin{tabular}{llllll}
\hline & $x$ & $y$ & $z$ & $U_{\text {iso }} * U_{\text {eq }}$ & Occ. $(<1)$ \\
\hline P1 & $0.30855(6)$ & $0.37650(5)$ & $0.09741(4)$ & $0.02340(16)$ & \\
P2 & $0.78163(6)$ & $0.24754(6)$ & $0.46477(4)$ & $0.02855(17)$ & \\
F1 & $0.42358(14)$ & $0.39964(13)$ & $0.03267(9)$ & $0.0368(4)$ & \\
F2 & $0.40636(15)$ & $0.46701(15)$ & $0.19023(9)$ & $0.0463(4)$ & \\
F3 & $0.23988(14)$ & $0.49934(12)$ & $0.07859(9)$ & $0.0342(3)$ & \\
F4 & $0.20934(16)$ & $0.28651(14)$ & $0.00513(10)$ & $0.0477(4)$ & \\
F5 & $0.37474(17)$ & $0.25367(14)$ & $0.11626(10)$ & $0.0485(4)$ & \\
F6 & $0.19177(15)$ & $0.35492(14)$ & $0.16311(10)$ & $0.0449(4)$ & \\
F7' & $0.8866(8)$ & $0.1865(7)$ & $0.5320(5)$ & $0.0485(9)$ & $0.592(6)$ \\
F8' & $0.8069(6)$ & $0.1427(5)$ & $0.3741(3)$ & $0.0583(12)$ & $0.592(6)$ \\
F9' & $0.6552(6)$ & $0.1530(5)$ & $0.4805(4)$ & $0.0444(11)$ & $0.592(6)$ \\
F10' & $0.7621(6)$ & $0.3542(5)$ & $0.5615(4)$ & $0.0595(14)$ & $0.592(6)$ \\
F11 & $0.9139(4)$ & $0.3438(5)$ & $0.4548(4)$ & $0.0648(13)$ & $0.592(6)$ \\
F12 & $0.6806(7)$ & $0.3126(7)$ & $0.4031(6)$ & $0.0322(4)$ & $0.592(6)$ \\
F7 & $0.8809(11)$ & $0.1709(11)$ & $0.5169(8)$ & $0.0485(9)$ & $0.408(6)$ \\
F8 & $0.7411(7)$ & $0.1201(7)$ & $0.3742(5)$ & $0.0583(12)$ & $0.408(6)$ \\
F9 & $0.6530(9)$ & $0.1932(7)$ & $0.5062(6)$ & $0.0444(11)$ & $0.408(6)$ \\
F10 & $0.8134(9)$ & $0.3697(7)$ & $0.5478(6)$ & $0.0595(14)$ & $0.408(6)$ \\
F11 & $0.9009(7)$ & $0.2907(8)$ & $0.4109(5)$ & $0.0648(13)$ & $0.408(6)$ \\
F12 & $0.6762(11)$ & $0.3186(11)$ & $0.4054(8)$ & $0.0322(4)$ & $0.408(6)$ \\
O1 & $1.00560(16)$ & $0.81093(14)$ & $0.22635(11)$ & $0.0328(4)$ & \\
O2 & $0.27675(18)$ & $0.27512(15)$ & $0.54849(10)$ & $0.0333(4)$ & \\
N1 & $0.85004(17)$ & $0.55784(16)$ & $0.20963(12)$ & $0.0205(4)$ & \\
& & & & &
\end{tabular}

Hydrogen site location: inferred from

$w=1 /\left[\sigma^{2}\left(F_{\mathrm{o}}^{2}\right)+(0.0518 P)^{2}\right]$

where $P=\left(F_{\mathrm{o}}{ }^{2}+2 F_{\mathrm{c}}{ }^{2}\right) / 3$

$(\Delta / \sigma)_{\max }=0.002$

$\Delta \rho_{\min }=-0.40 \mathrm{e} \AA^{-3}$

Extinction correction: SHELXS97 (Sheldrick,

2008), $\mathrm{Fc}^{*}=\mathrm{kFc}\left[1+0.001 \times \mathrm{xF}^{2} \lambda^{3} / \sin (2 \theta)\right]^{-1 / 4}$

Extinction coefficient: 0.0123 (11) 


\begin{tabular}{|c|c|c|c|c|}
\hline $\mathrm{N} 2$ & $0.23494(18)$ & $0.16209(16)$ & $0.34181(12)$ & $0.0215(4)$ \\
\hline $\mathrm{C} 1$ & $0.9767(2)$ & $0.5774(2)$ & $0.16140(15)$ & $0.0235(5)$ \\
\hline $\mathrm{H} 1 \mathrm{~A}$ & 0.9459 & 0.5845 & 0.0957 & $0.028 *$ \\
\hline H1B & 1.0272 & 0.5007 & 0.1559 & $0.028 *$ \\
\hline $\mathrm{C} 2$ & $1.0748(2)$ & $0.6979(2)$ & $0.21631(17)$ & $0.0295(5)$ \\
\hline $\mathrm{H} 2 \mathrm{~A}$ & 1.1104 & 0.6888 & 0.2807 & $0.035^{*}$ \\
\hline $\mathrm{H} 2 \mathrm{~B}$ & 1.1556 & 0.7076 & 0.1820 & $0.035^{*}$ \\
\hline $\mathrm{C} 3$ & $0.8918(2)$ & $0.8002(2)$ & $0.27847(16)$ & $0.0304(5)$ \\
\hline $\mathrm{H} 3 \mathrm{~A}$ & 0.8457 & 0.8797 & 0.2861 & $0.037 *$ \\
\hline H3B & 0.9276 & 0.7928 & 0.3433 & $0.037^{*}$ \\
\hline $\mathrm{C} 4$ & $0.7860(2)$ & $0.6831(2)$ & $0.22778(16)$ & $0.0262(5)$ \\
\hline $\mathrm{H} 4 \mathrm{~A}$ & 0.7435 & 0.6950 & 0.1657 & $0.031^{*}$ \\
\hline H4B & 0.7107 & 0.6769 & 0.2673 & $0.031 *$ \\
\hline $\mathrm{C} 5$ & $0.7400(2)$ & $0.4531(2)$ & $0.14237(15)$ & $0.0234(5)$ \\
\hline $\mathrm{H} 5 \mathrm{~A}$ & 0.6536 & 0.4513 & 0.1721 & $0.028 *$ \\
\hline $\mathrm{H} 5 \mathrm{~B}$ & 0.7180 & 0.4783 & 0.0817 & $0.028 *$ \\
\hline C6 & $0.7801(2)$ & $0.3181(2)$ & $0.11851(15)$ & $0.0237(5)$ \\
\hline $\mathrm{C} 7$ & $0.7297(2)$ & $0.2279(2)$ & $0.16439(17)$ & $0.0317(5)$ \\
\hline $\mathrm{H} 7$ & 0.6761 & 0.2540 & 0.2147 & $0.038^{*}$ \\
\hline $\mathrm{C} 8$ & $0.7564(3)$ & $0.1000(2)$ & 0.13772 (19) & $0.0406(6)$ \\
\hline $\mathrm{H} 8$ & 0.7215 & 0.0391 & 0.1697 & $0.049^{*}$ \\
\hline C9 & $0.8342(3)$ & $0.0617(2)$ & $0.0645(2)$ & $0.0433(7)$ \\
\hline H9 & 0.8526 & -0.0257 & 0.0457 & $0.052 *$ \\
\hline $\mathrm{C} 10$ & $0.8852(3)$ & $0.1513(3)$ & $0.01851(19)$ & $0.0415(7)$ \\
\hline $\mathrm{H} 10$ & 0.9394 & 0.1252 & -0.0314 & $0.050 *$ \\
\hline $\mathrm{C} 11$ & $0.8581(2)$ & $0.2777(2)$ & $0.04457(16)$ & $0.0314(6)$ \\
\hline H11 & 0.8927 & 0.3380 & 0.0120 & $0.038^{*}$ \\
\hline $\mathrm{C} 12$ & $0.8884(2)$ & $0.5197(2)$ & $0.30086(14)$ & $0.0262(5)$ \\
\hline $\mathrm{H} 12 \mathrm{~A}$ & 0.9362 & 0.4423 & 0.2874 & $0.039 *$ \\
\hline H12B & 0.8035 & 0.5008 & 0.3279 & $0.039 *$ \\
\hline $\mathrm{H} 12 \mathrm{C}$ & 0.9505 & 0.5912 & 0.3470 & $0.039 *$ \\
\hline $\mathrm{C} 13$ & $0.2326(2)$ & $0.3049(2)$ & 0.38787 (16) & $0.0275(5)$ \\
\hline $\mathrm{H} 13 \mathrm{~A}$ & 0.1351 & 0.3204 & 0.3943 & $0.033^{*}$ \\
\hline H13B & 0.2675 & 0.3568 & 0.3457 & $0.033^{*}$ \\
\hline $\mathrm{C} 14$ & $0.3215(3)$ & $0.3497(2)$ & $0.48603(16)$ & $0.0321(6)$ \\
\hline $\mathrm{H} 14 \mathrm{~A}$ & 0.4204 & 0.3410 & 0.4792 & $0.038^{*}$ \\
\hline H14B & 0.3151 & 0.4426 & 0.5144 & $0.038^{*}$ \\
\hline $\mathrm{C} 15$ & $0.2877(3)$ & $0.1415(2)$ & $0.50940(15)$ & $0.0297(5)$ \\
\hline $\mathrm{H} 15 \mathrm{~A}$ & 0.2595 & 0.0912 & 0.5543 & $0.036^{*}$ \\
\hline H15B & 0.3861 & 0.1314 & 0.5018 & $0.036^{*}$ \\
\hline $\mathrm{C} 16$ & $0.1962(2)$ & $0.0883(2)$ & $0.41301(15)$ & $0.0245(5)$ \\
\hline H16A & 0.2060 & -0.0045 & 0.3877 & $0.029^{*}$ \\
\hline $\mathrm{H} 16 \mathrm{~B}$ & 0.0972 & 0.0943 & 0.4212 & $0.029 *$ \\
\hline $\mathrm{C} 17$ & $0.3792(2)$ & $0.1439(2)$ & $0.31398(15)$ & $0.0233(5)$ \\
\hline H17A & 0.4005 & 0.2021 & 0.2728 & $0.028 *$ \\
\hline H17B & 0.4486 & 0.1724 & 0.3731 & $0.028 *$ \\
\hline $\mathrm{C} 18$ & $0.3991(2)$ & $0.0077(2)$ & $0.26190(15)$ & $0.0222(5)$ \\
\hline C19 & $0.4247(2)$ & $-0.0859(2)$ & $0.31083(15)$ & $0.0259(5)$ \\
\hline
\end{tabular}




\begin{tabular}{lllll}
$\mathrm{H} 19$ & 0.4245 & -0.0661 & 0.3782 & $0.031^{*}$ \\
$\mathrm{C} 20$ & $0.4506(2)$ & $-0.2080(2)$ & $0.26217(16)$ & $0.0305(5)$ \\
$\mathrm{H} 20$ & 0.4643 & -0.2723 & 0.2959 & $0.037^{*}$ \\
$\mathrm{C} 21$ & $0.4564(2)$ & $-0.2362(2)$ & $0.16470(16)$ & $0.0281(5)$ \\
$\mathrm{H} 21$ & 0.4756 & -0.3193 & 0.1316 & $0.034^{*}$ \\
$\mathrm{C} 22$ & $0.4341(2)$ & $-0.1434(2)$ & $0.11574(16)$ & $0.0294(5)$ \\
$\mathrm{H} 22$ & 0.4394 & -0.1622 & 0.0490 & $0.035^{*}$ \\
$\mathrm{C} 23$ & $0.4040(2)$ & $-0.0223(2)$ & $0.16372(15)$ & $0.0278(5)$ \\
$\mathrm{H} 23$ & 0.3866 & 0.0405 & 0.1292 & $0.033^{*}$ \\
$\mathrm{C} 24$ & $0.1262(2)$ & $0.1182(2)$ & $0.25385(15)$ & $0.0289(5)$ \\
$\mathrm{H} 24 \mathrm{~A}$ & 0.1221 & 0.0244 & 0.2269 & $0.043^{*}$ \\
$\mathrm{H} 24 \mathrm{~B}$ & 0.1508 & 0.1637 & 0.2061 & $0.043^{*}$ \\
$\mathrm{H} 24 \mathrm{C}$ & 0.0350 & 0.1380 & 0.2712 & $0.043^{*}$ \\
\hline
\end{tabular}

Atomic displacement parameters $\left(\AA^{2}\right)$

\begin{tabular}{|c|c|c|c|c|c|c|}
\hline & $U^{11}$ & $U^{22}$ & $U^{33}$ & $U^{12}$ & $U^{13}$ & $U^{23}$ \\
\hline $\mathrm{P} 1$ & $0.0243(3)$ & $0.0239(3)$ & $0.0254(3)$ & $0.0053(2)$ & $0.0057(3)$ & $0.0112(2)$ \\
\hline $\mathrm{P} 2$ & $0.0243(3)$ & $0.0329(4)$ & $0.0321(4)$ & $0.0022(3)$ & $0.0026(3)$ & $0.0165(3)$ \\
\hline $\mathrm{F} 1$ & $0.0325(8)$ & $0.0462(9)$ & $0.0401(8)$ & $0.0109(7)$ & $0.0181(7)$ & $0.0186(7)$ \\
\hline F2 & $0.0434(9)$ & $0.0533(10)$ & $0.0331(8)$ & $0.0054(7)$ & $-0.0054(7)$ & $0.0000(7)$ \\
\hline F3 & $0.0361(8)$ & $0.0347(8)$ & $0.0433(8)$ & $0.0166(6)$ & $0.0148(7)$ & $0.0229(6)$ \\
\hline F4 & $0.0494(10)$ & $0.0389(9)$ & $0.0444(9)$ & $-0.0092(7)$ & $-0.0088(7)$ & $0.0046(7)$ \\
\hline F5 & $0.0645(11)$ & $0.0424(9)$ & $0.0555(10)$ & $0.0321(8)$ & $0.0210(8)$ & $0.0288(8)$ \\
\hline F6 & $0.0417(9)$ & $0.0553(10)$ & $0.0563(10)$ & $0.0147(7)$ & $0.0240(8)$ & $0.0378(8)$ \\
\hline F7' & $0.0379(11)$ & 0.0567 (19) & $0.058(2)$ & $0.0078(12)$ & $-0.0078(12)$ & $0.0350(18)$ \\
\hline F8 & $0.076(3)$ & $0.070(2)$ & $0.0396(10)$ & $0.046(2)$ & $0.020(2)$ & $0.0151(12)$ \\
\hline F9' & $0.0339(9)$ & $0.047(3)$ & $0.057(3)$ & $-0.0077(18)$ & $0.0016(16)$ & $0.031(2)$ \\
\hline F10' & $0.084(4)$ & $0.0476(16)$ & $0.0360(16)$ & $0.016(2)$ & $-0.0128(19)$ & $-0.0014(13)$ \\
\hline F11' & $0.0255(12)$ & $0.083(3)$ & $0.101(4)$ & $-0.0102(18)$ & $0.000(2)$ & $0.065(3)$ \\
\hline F12' & $0.0292(8)$ & $0.0341(10)$ & $0.0355(8)$ & $0.0051(6)$ & $-0.0023(7)$ & $0.0162(7)$ \\
\hline F7 & $0.0379(11)$ & 0.0567 (19) & $0.058(2)$ & $0.0078(12)$ & $-0.0078(12)$ & $0.0350(18)$ \\
\hline F8 & $0.076(3)$ & $0.070(2)$ & $0.0396(10)$ & $0.046(2)$ & $0.020(2)$ & $0.0151(12)$ \\
\hline F9 & $0.0339(9)$ & $0.047(3)$ & $0.057(3)$ & -0.0077 (18) & $0.0016(16)$ & $0.031(2)$ \\
\hline F10 & $0.084(4)$ & $0.0476(16)$ & $0.0360(16)$ & $0.016(2)$ & $-0.0128(19)$ & $-0.0014(13)$ \\
\hline F11 & $0.0255(12)$ & $0.083(3)$ & $0.101(4)$ & $-0.0102(18)$ & $0.000(2)$ & $0.065(3)$ \\
\hline F12 & $0.0292(8)$ & $0.0341(10)$ & $0.0355(8)$ & $0.0051(6)$ & $-0.0023(7)$ & $0.0162(7)$ \\
\hline $\mathrm{O} 1$ & $0.0361(10)$ & $0.0228(9)$ & $0.0409(10)$ & $0.0034(7)$ & $0.0130(8)$ & $0.0079(7)$ \\
\hline $\mathrm{O} 2$ & $0.0499(11)$ & $0.0283(9)$ & $0.0239(8)$ & $0.0131(8)$ & $0.0112(8)$ & $0.0051(7)$ \\
\hline $\mathrm{N} 1$ & $0.0187(9)$ & $0.0237(10)$ & $0.0204(9)$ & $0.0049(7)$ & $0.0041(8)$ & $0.0067(7)$ \\
\hline $\mathrm{N} 2$ & $0.0240(10)$ & $0.0193(9)$ & $0.0218(9)$ & $0.0034(7)$ & $0.0019(8)$ & $0.0069(7)$ \\
\hline $\mathrm{C} 1$ & $0.0192(11)$ & $0.0264(12)$ & $0.0259(12)$ & $0.0048(9)$ & $0.0083(9)$ & $0.0057(9)$ \\
\hline $\mathrm{C} 2$ & $0.0245(13)$ & $0.0277(12)$ & $0.0353(13)$ & $0.0032(10)$ & $0.0090(10)$ & $0.0041(10)$ \\
\hline $\mathrm{C} 3$ & $0.0327(14)$ & $0.0276(12)$ & $0.0336(13)$ & $0.0102(10)$ & $0.0116(11)$ & $0.0073(10)$ \\
\hline $\mathrm{C} 4$ & $0.0249(12)$ & $0.0272(12)$ & $0.0296(12)$ & $0.0107(10)$ & $0.0064(10)$ & $0.0092(10)$ \\
\hline $\mathrm{C} 5$ & $0.0174(11)$ & $0.0311(12)$ & $0.0212(11)$ & $0.0019(9)$ & $0.0002(9)$ & $0.0076(9)$ \\
\hline C6 & $0.0173(11)$ & $0.0285(12)$ & $0.0222(11)$ & $-0.0007(9)$ & $-0.0019(9)$ & $0.0046(9)$ \\
\hline $\mathrm{C} 7$ & $0.0268(13)$ & $0.0339(13)$ & $0.0311(13)$ & $-0.0031(10)$ & $-0.0029(10)$ & $0.0086(10)$ \\
\hline
\end{tabular}


supporting information

\begin{tabular}{lllllll} 
C8 & $0.0378(15)$ & $0.0310(14)$ & $0.0484(16)$ & $-0.0052(11)$ & $-0.0098(13)$ & $0.0133(12)$ \\
C9 & $0.0311(15)$ & $0.0283(14)$ & $0.0589(18)$ & $0.0046(11)$ & $-0.0114(13)$ & $-0.0017(13)$ \\
C10 & $0.0254(14)$ & $0.0392(15)$ & $0.0483(16)$ & $0.0031(11)$ & $0.0012(12)$ & $-0.0075(13)$ \\
C11 & $0.0241(13)$ & $0.0338(13)$ & $0.0313(13)$ & $-0.0005(10)$ & $0.0035(10)$ & $0.0013(10)$ \\
C12 & $0.0279(13)$ & $0.0285(12)$ & $0.0220(11)$ & $0.0041(10)$ & $-0.0006(10)$ & $0.0080(9)$ \\
C13 & $0.0340(14)$ & $0.0200(11)$ & $0.0316(12)$ & $0.0092(10)$ & $0.0069(11)$ & $0.0091(9)$ \\
C14 & $0.0435(15)$ & $0.0215(12)$ & $0.0299(13)$ & $0.0066(11)$ & $0.0067(11)$ & $0.0027(10)$ \\
C15 & $0.0414(15)$ & $0.0279(13)$ & $0.0245(12)$ & $0.0118(11)$ & $0.0099(11)$ & $0.0102(10)$ \\
C16 & $0.0255(12)$ & $0.0256(12)$ & $0.0273(12)$ & $0.0053(9)$ & $0.0097(10)$ & $0.0123(9)$ \\
C17 & $0.0225(12)$ & $0.0244(11)$ & $0.0218(11)$ & $-0.0016(9)$ & $0.0035(9)$ & $0.0056(9)$ \\
C18 & $0.0188(11)$ & $0.0231(11)$ & $0.0223(11)$ & $-0.0005(9)$ & $0.0023(9)$ & $0.0035(9)$ \\
C19 & $0.0226(12)$ & $0.0334(13)$ & $0.0227(11)$ & $0.0084(10)$ & $0.0024(9)$ & $0.0078(10)$ \\
C20 & $0.0273(13)$ & $0.0316(13)$ & $0.0363(13)$ & $0.0118(10)$ & $0.0052(11)$ & $0.0129(11)$ \\
C21 & $0.0214(12)$ & $0.0256(12)$ & $0.0343(13)$ & $0.0048(9)$ & $0.0051(10)$ & $0.0014(10)$ \\
C22 & $0.0297(13)$ & $0.0318(13)$ & $0.0230(12)$ & $-0.0008(10)$ & $0.0051(10)$ & $0.0014(10)$ \\
C23 & $0.0320(13)$ & $0.0264(12)$ & $0.0241(12)$ & $0.0014(10)$ & $0.0031(10)$ & $0.0063(10)$ \\
C24 & $0.0279(13)$ & $0.0281(12)$ & $0.0287(12)$ & $-0.0015(10)$ & $-0.0056(10)$ & $0.0102(10)$ \\
& & & & & & \\
\hline
\end{tabular}

Geometric parameters $\left(\AA,{ }^{\circ}\right)$

\begin{tabular}{llll}
\hline P1-F1 & $1.5843(13)$ & $\mathrm{C} 5-\mathrm{H} 5 \mathrm{~B}$ & 0.9900 \\
$\mathrm{P} 1-\mathrm{F} 5$ & $1.5893(13)$ & $\mathrm{C} 6-\mathrm{C} 7$ & $1.386(3)$ \\
$\mathrm{P} 1-\mathrm{F} 4$ & $1.5910(14)$ & $\mathrm{C} 6-\mathrm{C} 11$ & $1.395(3)$ \\
$\mathrm{P} 1-\mathrm{F} 2$ & $1.5919(14)$ & $\mathrm{C} 7-\mathrm{C} 8$ & $1.389(3)$ \\
$\mathrm{P} 1-\mathrm{F} 3$ & $1.6005(13)$ & $\mathrm{C} 7-\mathrm{H} 7$ & 0.9500 \\
$\mathrm{P} 1-\mathrm{F} 6$ & $1.6035(14)$ & $\mathrm{C} 8-\mathrm{C} 9$ & $1.382(4)$ \\
$\mathrm{P} 2-\mathrm{F} 10$ & $1.523(6)$ & $\mathrm{C} 8-\mathrm{H} 8$ & 0.9500 \\
$\mathrm{P} 2-\mathrm{F} 8^{\prime}$ & $1.568(4)$ & $\mathrm{C} 9-\mathrm{C} 10$ & $1.384(4)$ \\
$\mathrm{P} 2-\mathrm{F} 11$ & $1.569(5)$ & $\mathrm{C} 9-\mathrm{H} 9$ & 0.9500 \\
$\mathrm{P} 2-\mathrm{F} 7$ & $1.572(6)$ & $\mathrm{C} 10-\mathrm{C} 11$ & $1.374(3)$ \\
$\mathrm{P} 2-\mathrm{F} 9$ & $1.573(6)$ & $\mathrm{C} 10-\mathrm{H} 10$ & 0.9500 \\
$\mathrm{P} 2-\mathrm{F} 12^{\prime}$ & $1.578(5)$ & $\mathrm{C} 11-\mathrm{H} 11$ & 0.9500 \\
$\mathrm{P} 2-\mathrm{F} 9^{\prime}$ & $1.590(4)$ & $\mathrm{C} 12-\mathrm{H} 12 \mathrm{~A}$ & 0.9800 \\
$\mathrm{P} 2-\mathrm{F} 11^{\prime}$ & $1.612(4)$ & $\mathrm{C} 12-\mathrm{H} 12 \mathrm{~B}$ & 0.9800 \\
$\mathrm{P} 2-\mathrm{F} 10^{\prime}$ & $1.623(4)$ & $\mathrm{C} 12-\mathrm{H} 12 \mathrm{C}$ & 0.9800 \\
$\mathrm{P} 2-\mathrm{F} 7^{\prime}$ & $1.624(4)$ & $\mathrm{C} 13-\mathrm{C} 14$ & $1.517(3)$ \\
$\mathrm{P} 2-\mathrm{F} 8$ & $1.624(6)$ & $\mathrm{C} 13-\mathrm{H} 13 \mathrm{~A}$ & 0.9900 \\
$\mathrm{P} 2-\mathrm{F} 12$ & $1.626(6)$ & $\mathrm{C} 13-\mathrm{H} 13 \mathrm{~B}$ & 0.9900 \\
$\mathrm{O} 1-\mathrm{C} 3$ & $1.425(3)$ & $\mathrm{C} 14-\mathrm{H} 14 \mathrm{~A}$ & 0.9900 \\
$\mathrm{O} 1-\mathrm{C} 2$ & $1.427(3)$ & $\mathrm{C} 14-\mathrm{H} 14 \mathrm{~B}$ & 0.9900 \\
$\mathrm{O} 2-\mathrm{C} 15$ & $1.424(2)$ & $\mathrm{C} 15-\mathrm{C} 16$ & $1.513(3)$ \\
$\mathrm{O} 2-\mathrm{C} 14$ & $1.427(3)$ & $\mathrm{C} 15-\mathrm{H} 15 \mathrm{~A}$ & 0.9900 \\
$\mathrm{~N} 1-\mathrm{C} 12$ & $1.498(3)$ & $\mathrm{C} 15-\mathrm{H} 15 \mathrm{~B}$ & 0.9900 \\
$\mathrm{~N} 1-\mathrm{C} 1$ & $1.510(2)$ & $\mathrm{C} 16-\mathrm{H} 16 \mathrm{~A}$ & 0.9900 \\
$\mathrm{~N} 1-\mathrm{C} 4$ & $1.513(3)$ & $\mathrm{C} 16-\mathrm{H} 16 \mathrm{~B}$ & 0.9900 \\
$\mathrm{~N} 1-\mathrm{C} 5$ & $1.528(3)$ & $\mathrm{C} 17-\mathrm{C} 18$ & $1.512(3)$ \\
$\mathrm{N} 2-\mathrm{C} 24$ & $1.503(3)$ & $\mathrm{C} 17-\mathrm{H} 17 \mathrm{~A}$ & 0.9900 \\
$\mathrm{~N} 2-\mathrm{C} 16$ & $1.509(3)$ & $\mathrm{C} 17-\mathrm{H} 17 \mathrm{~B}$ & 0.9900 \\
& & &
\end{tabular}




\begin{tabular}{|c|c|c|c|}
\hline $\mathrm{N} 2-\mathrm{C} 13$ & $1.515(3)$ & $\mathrm{C} 18-\mathrm{C} 19$ & $1.391(3)$ \\
\hline $\mathrm{N} 2-\mathrm{C} 17$ & $1.524(3)$ & $\mathrm{C} 18-\mathrm{C} 23$ & $1.391(3)$ \\
\hline $\mathrm{C} 1-\mathrm{C} 2$ & $1.512(3)$ & $\mathrm{C} 19-\mathrm{C} 20$ & $1.387(3)$ \\
\hline $\mathrm{C} 1-\mathrm{H} 1 \mathrm{~A}$ & 0.9900 & C19-H19 & 0.9500 \\
\hline $\mathrm{C} 1-\mathrm{H} 1 \mathrm{~B}$ & 0.9900 & $\mathrm{C} 20-\mathrm{C} 21$ & $1.383(3)$ \\
\hline $\mathrm{C} 2-\mathrm{H} 2 \mathrm{~A}$ & 0.9900 & $\mathrm{C} 20-\mathrm{H} 20$ & 0.9500 \\
\hline $\mathrm{C} 2-\mathrm{H} 2 \mathrm{~B}$ & 0.9900 & $\mathrm{C} 21-\mathrm{C} 22$ & $1.377(3)$ \\
\hline $\mathrm{C} 3-\mathrm{C} 4$ & $1.512(3)$ & $\mathrm{C} 21-\mathrm{H} 21$ & 0.9500 \\
\hline $\mathrm{C} 3-\mathrm{H} 3 \mathrm{~A}$ & 0.9900 & $\mathrm{C} 22-\mathrm{C} 23$ & $1.391(3)$ \\
\hline $\mathrm{C} 3-\mathrm{H} 3 \mathrm{~B}$ & 0.9900 & $\mathrm{C} 22-\mathrm{H} 22$ & 0.9500 \\
\hline $\mathrm{C} 4-\mathrm{H} 4 \mathrm{~A}$ & 0.9900 & $\mathrm{C} 23-\mathrm{H} 23$ & 0.9500 \\
\hline $\mathrm{C} 4-\mathrm{H} 4 \mathrm{~B}$ & 0.9900 & $\mathrm{C} 24-\mathrm{H} 24 \mathrm{~A}$ & 0.9800 \\
\hline $\mathrm{C} 5-\mathrm{C} 6$ & $1.503(3)$ & $\mathrm{C} 24-\mathrm{H} 24 \mathrm{~B}$ & 0.9800 \\
\hline $\mathrm{C} 5-\mathrm{H} 5 \mathrm{~A}$ & 0.9900 & $\mathrm{C} 24-\mathrm{H} 24 \mathrm{C}$ & 0.9800 \\
\hline $\mathrm{F} 1-\mathrm{P} 1-\mathrm{F} 5$ & $90.51(8)$ & $\mathrm{O} 1-\mathrm{C} 2-\mathrm{H} 2 \mathrm{~A}$ & 109.4 \\
\hline $\mathrm{F} 1-\mathrm{P} 1-\mathrm{F} 4$ & $89.97(8)$ & $\mathrm{C} 1-\mathrm{C} 2-\mathrm{H} 2 \mathrm{~A}$ & 109.4 \\
\hline $\mathrm{F} 5-\mathrm{P} 1-\mathrm{F} 4$ & $90.45(9)$ & $\mathrm{O} 1-\mathrm{C} 2-\mathrm{H} 2 \mathrm{~B}$ & 109.4 \\
\hline $\mathrm{F} 1-\mathrm{P} 1-\mathrm{F} 2$ & $90.56(8)$ & $\mathrm{C} 1-\mathrm{C} 2-\mathrm{H} 2 \mathrm{~B}$ & 109.4 \\
\hline $\mathrm{F} 5-\mathrm{P} 1-\mathrm{F} 2$ & $89.86(9)$ & $\mathrm{H} 2 \mathrm{~A}-\mathrm{C} 2-\mathrm{H} 2 \mathrm{~B}$ & 108.0 \\
\hline $\mathrm{F} 4-\mathrm{P} 1-\mathrm{F} 2$ & $179.38(8)$ & $\mathrm{O} 1-\mathrm{C} 3-\mathrm{C} 4$ & $111.43(17)$ \\
\hline $\mathrm{F} 1-\mathrm{P} 1-\mathrm{F} 3$ & $90.16(7)$ & $\mathrm{O} 1-\mathrm{C} 3-\mathrm{H} 3 \mathrm{~A}$ & 109.3 \\
\hline $\mathrm{F} 5-\mathrm{P} 1-\mathrm{F} 3$ & $179.23(8)$ & $\mathrm{C} 4-\mathrm{C} 3-\mathrm{H} 3 \mathrm{~A}$ & 109.3 \\
\hline $\mathrm{F} 4-\mathrm{P} 1-\mathrm{F} 3$ & $89.18(8)$ & $\mathrm{O} 1-\mathrm{C} 3-\mathrm{H} 3 \mathrm{~B}$ & 109.3 \\
\hline $\mathrm{F} 2-\mathrm{P} 1-\mathrm{F} 3$ & $90.50(8)$ & $\mathrm{C} 4-\mathrm{C} 3-\mathrm{H} 3 \mathrm{~B}$ & 109.3 \\
\hline $\mathrm{F} 1-\mathrm{P} 1-\mathrm{F} 6$ & $179.29(8)$ & $\mathrm{H} 3 \mathrm{~A}-\mathrm{C} 3-\mathrm{H} 3 \mathrm{~B}$ & 108.0 \\
\hline $\mathrm{F} 5-\mathrm{P} 1-\mathrm{F} 6$ & $90.15(8)$ & $\mathrm{C} 3-\mathrm{C} 4-\mathrm{N} 1$ & $112.51(18)$ \\
\hline $\mathrm{F} 4-\mathrm{P} 1-\mathrm{F} 6$ & $90.26(8)$ & $\mathrm{C} 3-\mathrm{C} 4-\mathrm{H} 4 \mathrm{~A}$ & 109.1 \\
\hline $\mathrm{F} 2-\mathrm{P} 1-\mathrm{F} 6$ & $89.20(8)$ & $\mathrm{N} 1-\mathrm{C} 4-\mathrm{H} 4 \mathrm{~A}$ & 109.1 \\
\hline $\mathrm{F} 3-\mathrm{P} 1-\mathrm{F} 6$ & $89.18(7)$ & $\mathrm{C} 3-\mathrm{C} 4-\mathrm{H} 4 \mathrm{~B}$ & 109.1 \\
\hline $\mathrm{F} 10-\mathrm{P} 2-\mathrm{F}^{\prime}$ & $157.7(3)$ & $\mathrm{N} 1-\mathrm{C} 4-\mathrm{H} 4 \mathrm{~B}$ & 109.1 \\
\hline $\mathrm{F} 10-\mathrm{P} 2-\mathrm{F} 11$ & $94.0(3)$ & $\mathrm{H} 4 \mathrm{~A}-\mathrm{C} 4-\mathrm{H} 4 \mathrm{~B}$ & 107.8 \\
\hline $\mathrm{F} 8{ }^{\prime}-\mathrm{P} 2-\mathrm{F} 11$ & $64.7(3)$ & $\mathrm{C} 6-\mathrm{C} 5-\mathrm{N} 1$ & $115.64(17)$ \\
\hline $\mathrm{F} 10-\mathrm{P} 2-\mathrm{F} 7$ & $93.1(4)$ & $\mathrm{C} 6-\mathrm{C} 5-\mathrm{H} 5 \mathrm{~A}$ & 108.4 \\
\hline $\mathrm{F} 8^{\prime}-\mathrm{P} 2-\mathrm{F} 7$ & $81.6(5)$ & $\mathrm{N} 1-\mathrm{C} 5-\mathrm{H} 5 \mathrm{~A}$ & 108.4 \\
\hline $\mathrm{F} 11-\mathrm{P} 2-\mathrm{F} 7$ & 91.5 (4) & $\mathrm{C} 6-\mathrm{C} 5-\mathrm{H} 5 \mathrm{~B}$ & 108.4 \\
\hline $\mathrm{F} 10-\mathrm{P} 2-\mathrm{F} 9$ & $92.6(3)$ & $\mathrm{N} 1-\mathrm{C} 5-\mathrm{H} 5 \mathrm{~B}$ & 108.4 \\
\hline $\mathrm{F} 8^{\prime}-\mathrm{P} 2-\mathrm{F} 9$ & $109.1(3)$ & $\mathrm{H} 5 \mathrm{~A}-\mathrm{C} 5-\mathrm{H} 5 \mathrm{~B}$ & 107.4 \\
\hline $\mathrm{F} 11-\mathrm{P} 2-\mathrm{F} 9$ & $172.8(4)$ & $\mathrm{C} 7-\mathrm{C} 6-\mathrm{C} 11$ & $118.6(2)$ \\
\hline $\mathrm{F} 7-\mathrm{P} 2-\mathrm{F} 9$ & $91.0(4)$ & $\mathrm{C} 7-\mathrm{C} 6-\mathrm{C} 5$ & $120.2(2)$ \\
\hline $\mathrm{F} 10-\mathrm{P} 2-\mathrm{F} 12^{\prime}$ & $92.7(6)$ & $\mathrm{C} 11-\mathrm{C} 6-\mathrm{C} 5$ & $120.9(2)$ \\
\hline $\mathrm{F} 8^{\prime}-\mathrm{P} 2-\mathrm{F} 12^{\prime}$ & $92.7(3)$ & $\mathrm{C} 6-\mathrm{C} 7-\mathrm{C} 8$ & $120.9(2)$ \\
\hline $\mathrm{F} 11-\mathrm{P} 2-\mathrm{F} 12^{\prime}$ & $87.2(5)$ & $\mathrm{C} 6-\mathrm{C} 7-\mathrm{H} 7$ & 119.6 \\
\hline $\mathrm{F} 7-\mathrm{P} 2-\mathrm{F} 12^{\prime}$ & $174.2(6)$ & $\mathrm{C} 8-\mathrm{C} 7-\mathrm{H} 7$ & 119.6 \\
\hline $\mathrm{F} 9-\mathrm{P} 2-\mathrm{F} 12^{\prime}$ & $89.6(6)$ & $\mathrm{C} 9-\mathrm{C} 8-\mathrm{C} 7$ & $119.7(3)$ \\
\hline $\mathrm{F} 10-\mathrm{P} 2-\mathrm{F} 9^{\prime}$ & $110.4(3)$ & $\mathrm{C} 9-\mathrm{C} 8-\mathrm{H} 8$ & 120.1 \\
\hline $\mathrm{F} 8^{\prime}-\mathrm{P} 2-\mathrm{F} 9^{\prime}$ & $91.0(2)$ & $\mathrm{C} 7-\mathrm{C} 8-\mathrm{H} 8$ & 120.1 \\
\hline $\mathrm{F} 11-\mathrm{P} 2-\mathrm{F}^{\prime}$ & $155.6(3)$ & $\mathrm{C} 8-\mathrm{C} 9-\mathrm{C} 10$ & $119.7(2)$ \\
\hline
\end{tabular}




\begin{tabular}{|c|c|c|c|}
\hline $\mathrm{F} 7-\mathrm{P} 2-\mathrm{F} 9^{\prime}$ & $86.9(6)$ & $\mathrm{C} 8-\mathrm{C} 9-\mathrm{H} 9$ & 120.1 \\
\hline $\mathrm{F} 9-\mathrm{P} 2-\mathrm{F} 9{ }^{\prime}$ & $18.2(3)$ & $\mathrm{C} 10-\mathrm{C} 9-\mathrm{H} 9$ & 120.1 \\
\hline $\mathrm{F} 12^{\prime}-\mathrm{P} 2-\mathrm{F} 9^{\prime}$ & $91.9(3)$ & $\mathrm{C} 11-\mathrm{C} 10-\mathrm{C} 9$ & $120.5(2)$ \\
\hline $\mathrm{F} 10-\mathrm{P} 2-\mathrm{F} 11^{\prime}$ & $67.6(3)$ & $\mathrm{C} 11-\mathrm{C} 10-\mathrm{H} 10$ & 119.7 \\
\hline $\mathrm{F} 8^{\prime}-\mathrm{P} 2-\mathrm{F} 11^{\prime}$ & 90.77 (19) & $\mathrm{C} 9-\mathrm{C} 10-\mathrm{H} 10$ & 119.7 \\
\hline $\mathrm{F} 11-\mathrm{P} 2-\mathrm{F} 11^{\prime}$ & $26.5(3)$ & $\mathrm{C} 10-\mathrm{C} 11-\mathrm{C} 6$ & $120.5(2)$ \\
\hline $\mathrm{F} 7-\mathrm{P} 2-\mathrm{F} 11^{\prime}$ & $90.7(5)$ & $\mathrm{C} 10-\mathrm{C} 11-\mathrm{H} 11$ & 119.7 \\
\hline $\mathrm{F} 9-\mathrm{P} 2-\mathrm{F} 11^{\prime}$ & $160.1(3)$ & $\mathrm{C} 6-\mathrm{C} 11-\mathrm{H} 11$ & 119.7 \\
\hline $\mathrm{F} 12^{\prime}-\mathrm{P} 2-\mathrm{F} 11^{\prime}$ & $90.7(3)$ & $\mathrm{N} 1-\mathrm{C} 12-\mathrm{H} 12 \mathrm{~A}$ & 109.5 \\
\hline $\mathrm{F} 9^{\prime}-\mathrm{P} 2-\mathrm{F} 11^{\prime}$ & $176.8(3)$ & $\mathrm{N} 1-\mathrm{C} 12-\mathrm{H} 12 \mathrm{~B}$ & 109.5 \\
\hline $\mathrm{F} 10-\mathrm{P} 2-\mathrm{F} 10^{\prime}$ & $21.1(4)$ & $\mathrm{H} 12 \mathrm{~A}-\mathrm{C} 12-\mathrm{H} 12 \mathrm{~B}$ & 109.5 \\
\hline $\mathrm{F} 8^{\prime}-\mathrm{P} 2-\mathrm{F} 10^{\prime}$ & $177.2(3)$ & $\mathrm{N} 1-\mathrm{C} 12-\mathrm{H} 12 \mathrm{C}$ & 109.5 \\
\hline $\mathrm{F} 11-\mathrm{P} 2-\mathrm{F} 10^{\prime}$ & $114.7(3)$ & $\mathrm{H} 12 \mathrm{~A}-\mathrm{C} 12-\mathrm{H} 12 \mathrm{C}$ & 109.5 \\
\hline $\mathrm{F} 7-\mathrm{P} 2-\mathrm{F} 10^{\prime}$ & $95.7(6)$ & $\mathrm{H} 12 \mathrm{~B}-\mathrm{C} 12-\mathrm{H} 12 \mathrm{C}$ & 109.5 \\
\hline $\mathrm{F} 9-\mathrm{P} 2-\mathrm{F} 10^{\prime}$ & $71.7(3)$ & $\mathrm{N} 2-\mathrm{C} 13-\mathrm{C} 14$ & $111.92(17)$ \\
\hline $\mathrm{F} 12^{\prime}-\mathrm{P} 2-\mathrm{F} 10^{\prime}$ & $90.0(3)$ & $\mathrm{N} 2-\mathrm{C} 13-\mathrm{H} 13 \mathrm{~A}$ & 109.2 \\
\hline $\mathrm{F} 9^{\prime}-\mathrm{P} 2-\mathrm{F} 10^{\prime}$ & $89.7(2)$ & $\mathrm{C} 14-\mathrm{C} 13-\mathrm{H} 13 \mathrm{~A}$ & 109.2 \\
\hline $\mathrm{F} 11^{\prime}-\mathrm{P} 2-\mathrm{F} 10^{\prime}$ & $88.5(2)$ & $\mathrm{N} 2-\mathrm{C} 13-\mathrm{H} 13 \mathrm{~B}$ & 109.2 \\
\hline $\mathrm{F} 10-\mathrm{P} 2-\mathrm{F} 7^{\prime}$ & $84.8(6)$ & $\mathrm{C} 14-\mathrm{C} 13-\mathrm{H} 13 \mathrm{~B}$ & 109.2 \\
\hline $\mathrm{F} 8^{\prime}-\mathrm{P} 2-\mathrm{F} 7^{\prime}$ & $89.7(3)$ & $\mathrm{H} 13 \mathrm{~A}-\mathrm{C} 13-\mathrm{H} 13 \mathrm{~B}$ & 107.9 \\
\hline $\mathrm{F} 11-\mathrm{P} 2-\mathrm{F}^{\prime}$ & $93.0(5)$ & $\mathrm{O} 2-\mathrm{C} 14-\mathrm{C} 13$ & $111.06(19)$ \\
\hline $\mathrm{F} 7-\mathrm{P} 2-\mathrm{F} 7{ }^{\prime}$ & $8.3(7)$ & $\mathrm{O} 2-\mathrm{C} 14-\mathrm{H} 14 \mathrm{~A}$ & 109.4 \\
\hline $\mathrm{F} 9-\mathrm{P} 2-\mathrm{F} 7^{\prime}$ & $90.4(6)$ & $\mathrm{C} 13-\mathrm{C} 14-\mathrm{H} 14 \mathrm{~A}$ & 109.4 \\
\hline $\mathrm{F} 12^{\prime}-\mathrm{P} 2-\mathrm{F} 7^{\prime}$ & $177.5(4)$ & $\mathrm{O} 2-\mathrm{C} 14-\mathrm{H} 14 \mathrm{~B}$ & 109.4 \\
\hline $\mathrm{F} 9^{\prime}-\mathrm{P} 2-\mathrm{F} 7^{\prime}$ & $88.9(3)$ & $\mathrm{C} 13-\mathrm{C} 14-\mathrm{H} 14 \mathrm{~B}$ & 109.4 \\
\hline $\mathrm{F} 11^{\prime}-\mathrm{P} 2-\mathrm{F} 7^{\prime}$ & $88.4(3)$ & $\mathrm{H} 14 \mathrm{~A}-\mathrm{C} 14-\mathrm{H} 14 \mathrm{~B}$ & 108.0 \\
\hline $\mathrm{F} 10^{\prime}-\mathrm{P} 2-\mathrm{F} 7^{\prime}$ & $87.6(3)$ & $\mathrm{O} 2-\mathrm{C} 15-\mathrm{C} 16$ & $111.24(17)$ \\
\hline $\mathrm{F} 10-\mathrm{P} 2-\mathrm{F} 8$ & $177.2(4)$ & $\mathrm{O} 2-\mathrm{C} 15-\mathrm{H} 15 \mathrm{~A}$ & 109.4 \\
\hline $\mathrm{F} 8^{\prime}-\mathrm{P} 2-\mathrm{F} 8$ & $23.8(2)$ & $\mathrm{C} 16-\mathrm{C} 15-\mathrm{H} 15 \mathrm{~A}$ & 109.4 \\
\hline $\mathrm{F} 11-\mathrm{P} 2-\mathrm{F} 8$ & $86.7(3)$ & $\mathrm{O} 2-\mathrm{C} 15-\mathrm{H} 15 \mathrm{~B}$ & 109.4 \\
\hline $\mathrm{F} 7-\mathrm{P} 2-\mathrm{F} 8$ & $89.6(4)$ & $\mathrm{C} 16-\mathrm{C} 15-\mathrm{H} 15 \mathrm{~B}$ & 109.4 \\
\hline $\mathrm{F} 9-\mathrm{P} 2-\mathrm{F} 8$ & $86.6(3)$ & $\mathrm{H} 15 \mathrm{~A}-\mathrm{C} 15-\mathrm{H} 15 \mathrm{~B}$ & 108.0 \\
\hline $\mathrm{F} 12^{\prime}-\mathrm{P} 2-\mathrm{F} 8$ & $84.6(5)$ & $\mathrm{N} 2-\mathrm{C} 16-\mathrm{C} 15$ & $111.46(18)$ \\
\hline $\mathrm{F} 9^{\prime}-\mathrm{P} 2-\mathrm{F} 8$ & $68.9(3)$ & $\mathrm{N} 2-\mathrm{C} 16-\mathrm{H} 16 \mathrm{~A}$ & 109.3 \\
\hline $\mathrm{F} 11^{\prime}-\mathrm{P} 2-\mathrm{F} 8$ & $113.2(2)$ & $\mathrm{C} 15-\mathrm{C} 16-\mathrm{H} 16 \mathrm{~A}$ & 109.3 \\
\hline $\mathrm{F} 10^{\prime}-\mathrm{P} 2-\mathrm{F} 8$ & $157.7(2)$ & $\mathrm{N} 2-\mathrm{C} 16-\mathrm{H} 16 \mathrm{~B}$ & 109.3 \\
\hline $\mathrm{F} 7{ }^{\prime}-\mathrm{P} 2-\mathrm{F} 8$ & $97.9(5)$ & $\mathrm{C} 15-\mathrm{C} 16-\mathrm{H} 16 \mathrm{~B}$ & 109.3 \\
\hline $\mathrm{F} 10-\mathrm{P} 2-\mathrm{F} 12$ & $90.5(4)$ & $\mathrm{H} 16 \mathrm{~A}-\mathrm{C} 16-\mathrm{H} 16 \mathrm{~B}$ & 108.0 \\
\hline $\mathrm{F} 8^{\prime}-\mathrm{P} 2-\mathrm{F} 12$ & $95.1(5)$ & $\mathrm{C} 18-\mathrm{C} 17-\mathrm{N} 2$ & $116.30(17)$ \\
\hline $\mathrm{F} 11-\mathrm{P} 2-\mathrm{F} 12$ & $88.5(4)$ & $\mathrm{C} 18-\mathrm{C} 17-\mathrm{H} 17 \mathrm{~A}$ & 108.2 \\
\hline $\mathrm{F} 7-\mathrm{P} 2-\mathrm{F} 12$ & $176.4(5)$ & $\mathrm{N} 2-\mathrm{C} 17-\mathrm{H} 17 \mathrm{~A}$ & 108.2 \\
\hline $\mathrm{F} 9-\mathrm{P} 2-\mathrm{F} 12$ & $88.6(4)$ & $\mathrm{C} 18-\mathrm{C} 17-\mathrm{H} 17 \mathrm{~B}$ & 108.2 \\
\hline $\mathrm{F} 12^{\prime}-\mathrm{P} 2-\mathrm{F} 12$ & $2.4(7)$ & $\mathrm{N} 2-\mathrm{C} 17-\mathrm{H} 17 \mathrm{~B}$ & 108.2 \\
\hline $\mathrm{F} 9{ }^{\prime}-\mathrm{P} 2-\mathrm{F} 12$ & $91.7(6)$ & $\mathrm{H} 17 \mathrm{~A}-\mathrm{C} 17-\mathrm{H} 17 \mathrm{~B}$ & 107.4 \\
\hline $\mathrm{F} 11^{\prime}-\mathrm{P} 2-\mathrm{F} 12$ & $90.9(5)$ & $\mathrm{C} 19-\mathrm{C} 18-\mathrm{C} 23$ & $118.67(19)$ \\
\hline $\mathrm{F} 10^{\prime}-\mathrm{P} 2-\mathrm{F} 12$ & $87.6(6)$ & $\mathrm{C} 19-\mathrm{C} 18-\mathrm{C} 17$ & $121.67(19)$ \\
\hline $\mathrm{F} 7{ }^{\prime}-\mathrm{P} 2-\mathrm{F} 12$ & $175.2(6)$ & $\mathrm{C} 23-\mathrm{C} 18-\mathrm{C} 17$ & $119.42(19)$ \\
\hline $\mathrm{F} 8-\mathrm{P} 2-\mathrm{F} 12$ & $86.8(4)$ & $\mathrm{C} 20-\mathrm{C} 19-\mathrm{C} 18$ & $120.6(2)$ \\
\hline
\end{tabular}




\begin{tabular}{|c|c|c|c|}
\hline $\mathrm{C} 3-\mathrm{O} 1-\mathrm{C} 2$ & $110.05(17)$ & $\mathrm{C} 20-\mathrm{C} 19-\mathrm{H} 19$ & 119.7 \\
\hline $\mathrm{C} 15-\mathrm{O} 2-\mathrm{C} 14$ & $110.09(15)$ & $\mathrm{C} 18-\mathrm{C} 19-\mathrm{H} 19$ & 119.7 \\
\hline $\mathrm{C} 12-\mathrm{N} 1-\mathrm{C} 1$ & $111.17(16)$ & $\mathrm{C} 21-\mathrm{C} 20-\mathrm{C} 19$ & $120.1(2)$ \\
\hline $\mathrm{C} 12-\mathrm{N} 1-\mathrm{C} 4$ & $111.53(16)$ & $\mathrm{C} 21-\mathrm{C} 20-\mathrm{H} 20$ & 119.9 \\
\hline $\mathrm{C} 1-\mathrm{N} 1-\mathrm{C} 4$ & $107.61(16)$ & $\mathrm{C} 19-\mathrm{C} 20-\mathrm{H} 20$ & 119.9 \\
\hline $\mathrm{C} 12-\mathrm{N} 1-\mathrm{C} 5$ & $109.33(16)$ & $\mathrm{C} 22-\mathrm{C} 21-\mathrm{C} 20$ & $119.8(2)$ \\
\hline $\mathrm{C} 1-\mathrm{N} 1-\mathrm{C} 5$ & $110.28(15)$ & $\mathrm{C} 22-\mathrm{C} 21-\mathrm{H} 21$ & 120.1 \\
\hline $\mathrm{C} 4-\mathrm{N} 1-\mathrm{C} 5$ & $106.81(16)$ & $\mathrm{C} 20-\mathrm{C} 21-\mathrm{H} 21$ & 120.1 \\
\hline $\mathrm{C} 24-\mathrm{N} 2-\mathrm{C} 16$ & $108.23(17)$ & $\mathrm{C} 21-\mathrm{C} 22-\mathrm{C} 23$ & $120.2(2)$ \\
\hline $\mathrm{C} 24-\mathrm{N} 2-\mathrm{C} 13$ & $109.28(16)$ & $\mathrm{C} 21-\mathrm{C} 22-\mathrm{H} 22$ & 119.9 \\
\hline $\mathrm{C} 16-\mathrm{N} 2-\mathrm{C} 13$ & $107.35(15)$ & $\mathrm{C} 23-\mathrm{C} 22-\mathrm{H} 22$ & 119.9 \\
\hline $\mathrm{C} 24-\mathrm{N} 2-\mathrm{C} 17$ & $109.83(16)$ & $\mathrm{C} 22-\mathrm{C} 23-\mathrm{C} 18$ & $120.5(2)$ \\
\hline $\mathrm{C} 16-\mathrm{N} 2-\mathrm{C} 17$ & $113.36(16)$ & $\mathrm{C} 22-\mathrm{C} 23-\mathrm{H} 23$ & 119.8 \\
\hline $\mathrm{C} 13-\mathrm{N} 2-\mathrm{C} 17$ & $108.70(16)$ & $\mathrm{C} 18-\mathrm{C} 23-\mathrm{H} 23$ & 119.8 \\
\hline $\mathrm{N} 1-\mathrm{C} 1-\mathrm{C} 2$ & $112.04(16)$ & $\mathrm{N} 2-\mathrm{C} 24-\mathrm{H} 24 \mathrm{~A}$ & 109.5 \\
\hline $\mathrm{N} 1-\mathrm{C} 1-\mathrm{H} 1 \mathrm{~A}$ & 109.2 & $\mathrm{~N} 2-\mathrm{C} 24-\mathrm{H} 24 \mathrm{~B}$ & 109.5 \\
\hline $\mathrm{C} 2-\mathrm{C} 1-\mathrm{H} 1 \mathrm{~A}$ & 109.2 & $\mathrm{H} 24 \mathrm{~A}-\mathrm{C} 24-\mathrm{H} 24 \mathrm{~B}$ & 109.5 \\
\hline $\mathrm{N} 1-\mathrm{C} 1-\mathrm{H} 1 \mathrm{~B}$ & 109.2 & $\mathrm{~N} 2-\mathrm{C} 24-\mathrm{H} 24 \mathrm{C}$ & 109.5 \\
\hline $\mathrm{C} 2-\mathrm{C} 1-\mathrm{H} 1 \mathrm{~B}$ & 109.2 & $\mathrm{H} 24 \mathrm{~A}-\mathrm{C} 24-\mathrm{H} 24 \mathrm{C}$ & 109.5 \\
\hline $\mathrm{H} 1 \mathrm{~A}-\mathrm{C} 1-\mathrm{H} 1 \mathrm{~B}$ & 107.9 & $\mathrm{H} 24 \mathrm{~B}-\mathrm{C} 24-\mathrm{H} 24 \mathrm{C}$ & 109.5 \\
\hline $\mathrm{O} 1-\mathrm{C} 2-\mathrm{C} 1$ & $110.99(18)$ & & \\
\hline $\mathrm{C} 12-\mathrm{N} 1-\mathrm{C} 1-\mathrm{C} 2$ & $-70.4(2)$ & $\mathrm{C} 24-\mathrm{N} 2-\mathrm{C} 13-\mathrm{C} 14$ & $170.01(18)$ \\
\hline $\mathrm{C} 4-\mathrm{N} 1-\mathrm{C} 1-\mathrm{C} 2$ & $52.0(2)$ & $\mathrm{C} 16-\mathrm{N} 2-\mathrm{C} 13-\mathrm{C} 14$ & $52.8(2)$ \\
\hline $\mathrm{C} 5-\mathrm{N} 1-\mathrm{C} 1-\mathrm{C} 2$ & $168.14(17)$ & $\mathrm{C} 17-\mathrm{N} 2-\mathrm{C} 13-\mathrm{C} 14$ & $-70.1(2)$ \\
\hline $\mathrm{C} 3-\mathrm{O} 1-\mathrm{C} 2-\mathrm{C} 1$ & $61.1(2)$ & $\mathrm{C} 15-\mathrm{O} 2-\mathrm{C} 14-\mathrm{C} 13$ & $60.1(2)$ \\
\hline $\mathrm{N} 1-\mathrm{C} 1-\mathrm{C} 2-\mathrm{O} 1$ & $-58.5(2)$ & $\mathrm{N} 2-\mathrm{C} 13-\mathrm{C} 14-\mathrm{O} 2$ & $-57.5(2)$ \\
\hline $\mathrm{C} 2-\mathrm{O} 1-\mathrm{C} 3-\mathrm{C} 4$ & $-60.1(2)$ & $\mathrm{C} 14-\mathrm{O} 2-\mathrm{C} 15-\mathrm{C} 16$ & $-61.0(2)$ \\
\hline $\mathrm{O} 1-\mathrm{C} 3-\mathrm{C} 4-\mathrm{N} 1$ & $56.6(2)$ & $\mathrm{C} 24-\mathrm{N} 2-\mathrm{C} 16-\mathrm{C} 15$ & $-171.17(16)$ \\
\hline $\mathrm{C} 12-\mathrm{N} 1-\mathrm{C} 4-\mathrm{C} 3$ & $71.1(2)$ & $\mathrm{C} 13-\mathrm{N} 2-\mathrm{C} 16-\mathrm{C} 15$ & $-53.3(2)$ \\
\hline $\mathrm{C} 1-\mathrm{N} 1-\mathrm{C} 4-\mathrm{C} 3$ & $-51.1(2)$ & $\mathrm{C} 17-\mathrm{N} 2-\mathrm{C} 16-\mathrm{C} 15$ & $66.7(2)$ \\
\hline $\mathrm{C} 5-\mathrm{N} 1-\mathrm{C} 4-\mathrm{C} 3$ & $-169.51(17)$ & $\mathrm{O} 2-\mathrm{C} 15-\mathrm{C} 16-\mathrm{N} 2$ & $59.0(2)$ \\
\hline $\mathrm{C} 12-\mathrm{N} 1-\mathrm{C} 5-\mathrm{C} 6$ & $-56.4(2)$ & $\mathrm{C} 24-\mathrm{N} 2-\mathrm{C} 17-\mathrm{C} 18$ & $-57.3(2)$ \\
\hline $\mathrm{C} 1-\mathrm{N} 1-\mathrm{C} 5-\mathrm{C} 6$ & $66.1(2)$ & $\mathrm{C} 16-\mathrm{N} 2-\mathrm{C} 17-\mathrm{C} 18$ & $63.9(2)$ \\
\hline $\mathrm{C} 4-\mathrm{N} 1-\mathrm{C} 5-\mathrm{C} 6$ & $-177.22(17)$ & $\mathrm{C} 13-\mathrm{N} 2-\mathrm{C} 17-\mathrm{C} 18$ & $-176.85(17)$ \\
\hline $\mathrm{N} 1-\mathrm{C} 5-\mathrm{C} 6-\mathrm{C} 7$ & $101.2(2)$ & $\mathrm{N} 2-\mathrm{C} 17-\mathrm{C} 18-\mathrm{C} 19$ & $-80.6(2)$ \\
\hline $\mathrm{N} 1-\mathrm{C} 5-\mathrm{C} 6-\mathrm{C} 11$ & $-84.4(2)$ & $\mathrm{N} 2-\mathrm{C} 17-\mathrm{C} 18-\mathrm{C} 23$ & $105.1(2)$ \\
\hline $\mathrm{C} 11-\mathrm{C} 6-\mathrm{C} 7-\mathrm{C} 8$ & $0.2(3)$ & $\mathrm{C} 23-\mathrm{C} 18-\mathrm{C} 19-\mathrm{C} 20$ & $-1.9(3)$ \\
\hline $\mathrm{C} 5-\mathrm{C} 6-\mathrm{C} 7-\mathrm{C} 8$ & $174.8(2)$ & $\mathrm{C} 17-\mathrm{C} 18-\mathrm{C} 19-\mathrm{C} 20$ & $-176.3(2)$ \\
\hline $\mathrm{C} 6-\mathrm{C} 7-\mathrm{C} 8-\mathrm{C} 9$ & $0.0(4)$ & $\mathrm{C} 18-\mathrm{C} 19-\mathrm{C} 20-\mathrm{C} 21$ & $2.5(3)$ \\
\hline $\mathrm{C} 7-\mathrm{C} 8-\mathrm{C} 9-\mathrm{C} 10$ & $0.2(4)$ & $\mathrm{C} 19-\mathrm{C} 20-\mathrm{C} 21-\mathrm{C} 22$ & $-1.1(3)$ \\
\hline $\mathrm{C} 8-\mathrm{C} 9-\mathrm{C} 10-\mathrm{C} 11$ & $-0.6(4)$ & $\mathrm{C} 20-\mathrm{C} 21-\mathrm{C} 22-\mathrm{C} 23$ & $-0.9(3)$ \\
\hline $\mathrm{C} 9-\mathrm{C} 10-\mathrm{C} 11-\mathrm{C} 6$ & $0.8(4)$ & $\mathrm{C} 21-\mathrm{C} 22-\mathrm{C} 23-\mathrm{C} 18$ & $1.5(3)$ \\
\hline $\mathrm{C} 7-\mathrm{C} 6-\mathrm{C} 11-\mathrm{C} 10$ & $-0.6(3)$ & $\mathrm{C} 19-\mathrm{C} 18-\mathrm{C} 23-\mathrm{C} 22$ & $-0.1(3)$ \\
\hline $\mathrm{C} 5-\mathrm{C} 6-\mathrm{C} 11-\mathrm{C} 10$ & $-175.1(2)$ & $\mathrm{C} 17-\mathrm{C} 18-\mathrm{C} 23-\mathrm{C} 22$ & $174.43(19)$ \\
\hline
\end{tabular}

